\title{
Transforming Cancer Indicators Begs Bold New Strategies from Biotechnology
}

\author{
By Agustín Lage, MD, PhD
}

A national cancer program is essentially a population health initiative that goes beyond satisfying the demand for health services to propose modifying specific objective indicators in a defined population through interventions that have a scientifically grounded probability of success.

In the case of malignant neoplasms, the three "gold standard" indicators are age-adjusted specific mortality, age-adjusted incidence, and average 5- or 10-year survival rates. All other frequently used indicators-such as stage distribution at time of diagnosis, participation in early diagnostic interventions, intensity of public health education efforts, and coverage of chemo- and radiotherapy services, among others-are valid to the extent they are predictors of the three "gold standard" indicators.

It is important to emphasize that these are, first of all, population indicators describing the health status of population groups. Second, they are objective and therefore not easily susceptible to subjective appraisals; and, third, they are oriented toward measuring long-term results rather than immediate outcomes of health interventions. Consequently, these indicators are stricter than those we use to determine if a certain diagnostic test is efficiently predictive, or if a certain therapy improves the health of an individual patient. If a diagnostic technology (magnetic resonance imaging, positron emission tomography, or a certain biochemical marker, for example) is tested and found to give doctors useful information, or if a certain therapy is shown to modify the clinical course of a disease better than previous therapies, then such findings fully justify introducing the technology or the therapy in medical practice. But this conclusion does not automatically transfer to a population health context. There is no guarantee that these interventions-although useful for treating a patient's concrete problem-can modify incidence, mortality or survival indicators. Transposing impact to the population level is a more difficult test. Can biotechnology pass? With which products and in which concrete medical situations? This is the subject at hand.

\section{Biotechnology Production}

Biotechnology is essentially a new manufacturing process. All manufacturing, in any industrial sector, is a transformative process in which raw materials are converted to final products through specific intermediate steps. What distinguishes biotechnology is that these intermediate steps occur inside a living cell used as a manufacturing plant. Acting as small factories, the cells proliferate and produce through fermentation. This was an old idea that merged with another-the deliberate transformation of cells' genetic information, "genetic engineering"-creating explo- sively new potential. Modern biotechnology thus emerged at the end of the 1970s from the fusion of genetic engineering and the fermentation technologies of biochemical engineering (Figure 1).

Despite its short history, the industry has taken off, boosted by its enormous potential. Consider just the diversity of imaginable products: we humans have over 30,000 genes and over one billion different antibody molecules, each of which could become a "product" of a biotechnology enterprise. Add bacterial products, many of which could have practical applications, and keep in mind that the biosphere contains more than 100 million different species of bacteria, each one with several thousand genes: the numbers approach our intuitive notion of infinite. In several countries, biotechnology production has emerged as a new branch of industry, since the capital investment and energy costs for setting up production lines are relatively low compared to other industrial sectors.

Since the first biotechnology company, Genentech, was founded in California in 1977, over 4,000 have appeared, $34 \%$ in the United States, 38\% in Europe, and the rest distributed in a few other countries. These companies employ over 200,000 workers, generate more than US $\$ 50$ billion in sales, and invest over US\$27 billion in research annually.[1,2] Although, in principle, biotechnology has multiple applications, more than $50 \%$ of companies and over $90 \%$ of total research-anddevelopment spending are devoted to human health applications. Globally, the biotechnology industry has produced over 125 new drugs, and at least 600 more are in different phases of clinical trials.[3]

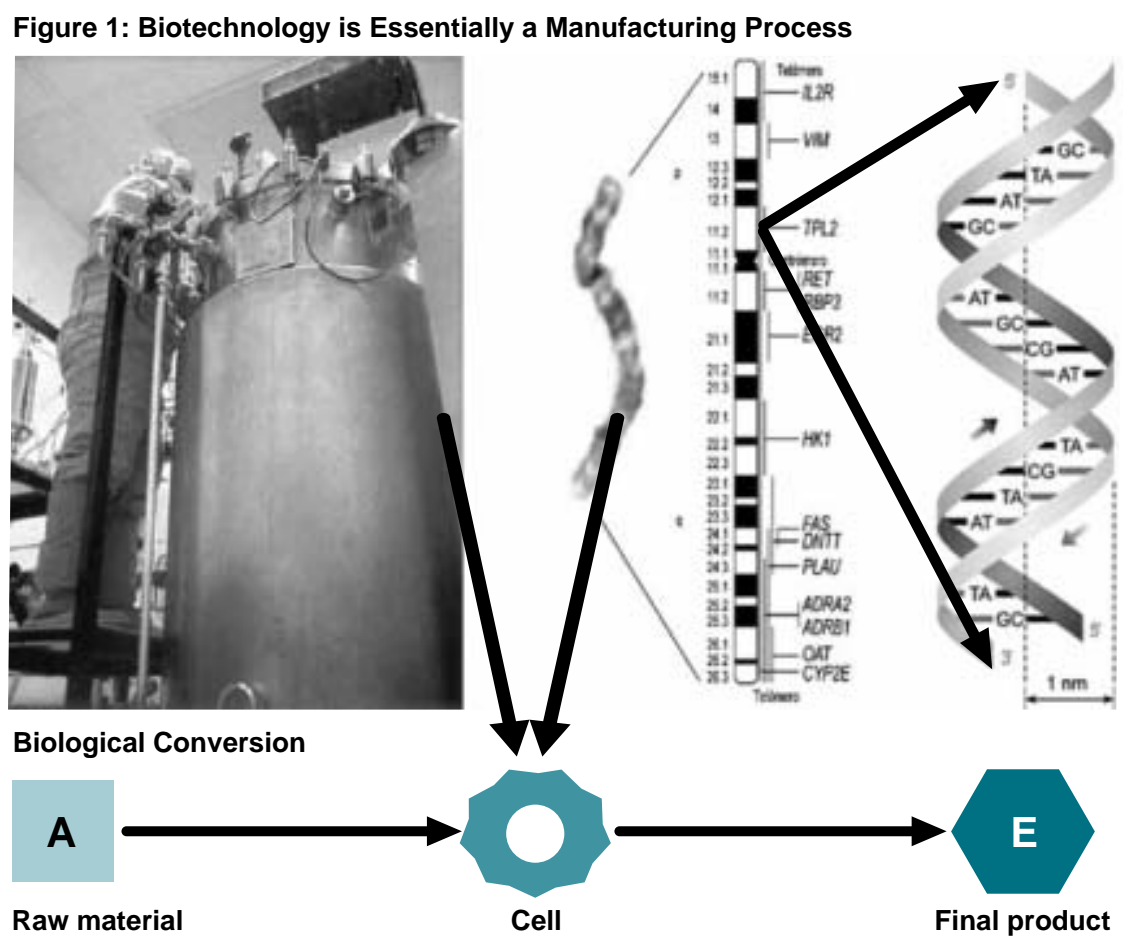




\section{Biotechnology and Cancer}

Although biotechnology's greatest therapeutic successes have been replacement therapy products, such as insulin and erythropoietin, the top research priority has always been treatment of malignant neoplasms: more than $50 \%$ of all product research worldwide falls in this category. Despite this global effort, however, the performance of biotechnology research in cancer treatment has thus far been less than brilliant. Recombinant interferon is certainly useful for treating some malignant neoplasms, such as melanoma, renal carcinoma and some forms of leukemia, but neoplasms causing the greatest share of cancer mortality (lung, breast, prostate, uterus and colon) are resistant to these treatments.

Monoclonal antibodies have been introduced in oncology practice in recent years on the basis of proven, but small, clinical benefits. Erbitux (chimeric antibody targeting the epidermal growth factor receptor) obtained a $10 \%$ objective response in colon cancer patients. In their respective clinical trials, Herceptin antibodies (for breast cancer) and Avastin (for colon cancer) produced survival advantages of slightly over five months. Although there are more than 50 therapeutic cancer vaccines in different phases of clinical trials, none has yet entered medical practice.

The scientific explanations for this dissociation between efforts and results are open to debate. Certainly there are essential biological sciences problems that need to be addressed, such as genetic control of cell proliferation and senescence mechanisms in the immune system. But there is another factor influencing this situation, which is socioeconomic. The vast majority of scientific research is financed and carried out by competing biotechnology and pharmaceutical companies focused on short-term market gains.

The consequences are many and diverse, but one is that products of cancer treatment research are evaluated for their "objective response," that is, their effect on short-term reduction in tumor size. We know today that objective response is not a good predictor of long-term survival. Treatments that

Figure 2: Cuban Biotechnology Products Targeting Cancer

\begin{tabular}{|c|c|c|}
\hline Product & Lead Institution & Application \\
\hline Recombinant Hepatitis B vaccine & $\begin{array}{l}\text { Center for Genetic Engineering and } \\
\text { Biotechnology (CIGB) }\end{array}$ & $\begin{array}{l}\text { Although not initially conceived as an oncology product, it is leading to } \\
\text { elimination of hepatitis B in the young Cuban population, which should } \\
\text { make a predictable impact on reducing the incidence of liver cancer. }\end{array}$ \\
\hline Prostate Specific Antigen (PSA) & Immunoassay Center (CIE) & Should permit earlier diagnosis of prostate cancer. \\
\hline Interferon & $\begin{array}{l}\text { Center for Genetic Engineering and } \\
\text { Biotechnology (CIGB) }\end{array}$ & Treatment of neoplasms for which it is indicated. \\
\hline Colony Stimulating Factor (CSF) & $\begin{array}{l}\text { Center for Molecular Immunology } \\
\text { (CIM) }\end{array}$ & $\begin{array}{l}\text { Treatment of chemotherapy-induced neutropenia, already helping im- } \\
\text { prove the efficacy of several cancer treatments, particularly in pediatric } \\
\text { oncology. }\end{array}$ \\
\hline Nimutozumab Monoclonal Antibody & $\begin{array}{l}\text { Center for Molecular Immunology } \\
\text { (CIM) }\end{array}$ & $\begin{array}{l}\text { Treatment of brain tumors, and head and neck tumors. Currently be- } \\
\text { ing evaluated for treatment of eight other neoplasms, including lung, } \\
\text { breast, uterine, prostate, and digestive tract tumors. }\end{array}$ \\
\hline EGF Vaccine & $\begin{array}{l}\text { Center for Molecular Immunology } \\
\text { (CIM) }\end{array}$ & $\begin{array}{l}\text { First therapeutic vaccine, registered in } 2008 \text { based on results showing } \\
\text { increased survival in advanced lung cancer patients. This vaccine is } \\
\text { also in prostate cancer clinical trials. }\end{array}$ \\
\hline Others & & $\begin{array}{l}\text { In addition to registered products, anticancer products in Cuba's biotech- } \\
\text { nology pipeline include seven monoclonal antibodies and six therapeutic } \\
\text { vaccines in different phases of preclinical and clinical research (Figure 3). }\end{array}$ \\
\hline
\end{tabular}


for mass use is consistent with the Cuban health system's preventive approach and is one of the distinctive features of Cuban biotechnology compared to the global industry.

At the same time, a major effort is being made to develop biotechnology applications for control of malignant neoplasms. This includes three components, the first of which is obtaining new products. Cuban biotechnology's current lines of cancerrelated products (cytokines, monoclonal antibodies and vaccines) differ little from those of its peers in other countries (Figure 3).

However, the other two components distinguish the $\mathrm{Cu}$ ban industry: its integration into the health system and a population approach through the national Comprehensive Cancer Control Program.

\section{Biotechnology and the National Cancer Program}

Cuba's organized social response to the cancer challenge has gone through four stages:

- The foundation was laid in the 1960s and 1970s. In 1964, the National Cancer Registry was created, and the National Oncology and Radiobiology Institute was founded in 1966. The national network of oncological units was also set up, guaranteeing radio and chemotherapy throughout Cuba. Oncology was established as a medical specialty. Mass screening for cervical cancer began in 1967.

- $\quad$ During the 1980s, the Comprehensive Program for Reduction of Cancer Mortality was created, bringing together available human and material resources in a strategy aimed at controlling cancer, through organized initiatives in every province.

- The economic crisis of the 1990s, called the "special period" in Cuba, curtailed the deployment of the potential built up in the preceding decades. Nevertheless, during this stage primary health care services were strengthened and expanded, as was the Scientific Pole.

- The current stage is marked by the creation of the $\mathrm{Na}$ tional Cancer Control Unit in 2006 in the Ministry of Public Health (MINSAP), and the restructuring of the national cancer program in the context of new conditions: 1) the high ratio of doctors and nurses per inhabitant and reinforced primary health care; 2) Cuba's economic recovery and investment process in hospitals and medical technology; and 3) existence of the pharmaceutical and biotechnology industry.
Figure 3: Pipeline of Cancer Products in Cuban Biotechnology

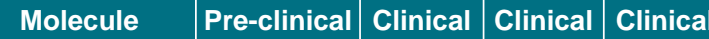
Characterization $\begin{gathered}\text { Evaluation } \\ \text { Trial (I) }\end{gathered}$

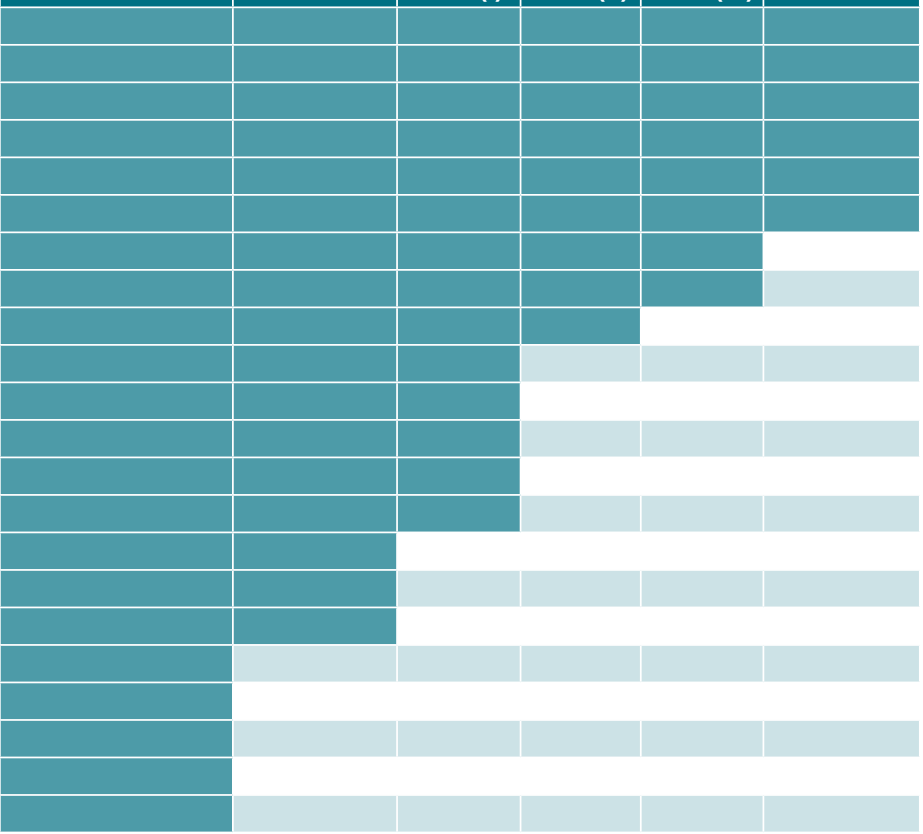

The national Comprehensive Cancer Control Program is much more than a program for improving oncological care (although this is one part). It is an organized effort aimed at modifying the "hard indicators"-incidence, mortality and survival. It includes initiatives at all levels of the health system, as well as multisector actions involving the educational system, local governments, social science institutes, social and political organizations, and other actors in Cuban society. It also includes a new component not found in the earlier stages of the Cuban Cancer Program: the impact of biotechnology.

Can biotechnology transcend its present (evident, but limited) role in the treatment of cancer patients and contribute to the modification of population health indicators? The unequivocal answer is, yes, it can. But part of the answer also lies in the conviction that impact will not come about spontaneously, but will require a directed strategy that goes beyond research and production of new products.

Let's look at what is needed for such a strategy to crystallize:

- The first requirement is to have products and the installed capacity to produce them. This means making products available to the whole population, not just "on the market". This cannot be accomplished by importing finished medicines at current multinational pharmaceutical prices. But having products is only the starting point. The ideas that follow concern the strategy for research and utilization of those products, and integration of the biotechnology sector with the public health system.

- The capacity and practice of clinical trials must be (exponentially) expanded throughout the health system. This is a very important conceptual change, suggesting that clinical trials must include but go beyond scientific evaluation of new products. Extending the methodology and practice 
of clinical trials to all cancer-care institutions introduces in the health system the capacity to continuously evaluate the population impact of everything being done. It also extends a culture of rigor and systematization in therapeutic administration and record-keeping.

Clinical research includes a range of objectives and methodologies. The typical Phase 1,2 and 3 trial sequence, with restrictive inclusion criteria for evaluating new products, remains at one end of the spectrum; at the other end is prospective observation of everything done in medical practice and construction of databases that accumulate real-time information on therapeutic outcomes. Between these two lies a range of trial types, including randomized trials with broader inclusion criteria, and expanded access programs (EAPs) for products in different stages of evaluation, among others. The goal of expanded trials has not yet been achieved, but advances are being made: more than 50 clinical trials with biotechnology products are currently underway in Cuba, involving some 3,000 patients and over 350 physicians in 52 hospitals.

- Priority should be given to clinical trials of chronic therapeutic interventions with survival as the final evaluation criteria. A potential advantage of biotechnology products for cancer treatment is their low toxicity, facilitating their use over a prolonged period. Although the Program includes crucial efforts aimed at early detection and primary prevention, many neoplasms are not vulnerable to this type of intervention, nor would it be ethical to make the patient responsible for consulting the doctor "too late."

Current incidence and mortality rates indicate that at least one out of every three people will suffer some form of cancer at some time in their life, and at least half will reach the advanced stage of the disease at some point. Advanced cancer is and will be an important target for the health system, for which a population strategy should also be adopted. The goal here should be the step-by-step transformation of advanced cancer into a chronic disease by affecting its clinical course (Figure 4). As such, and like other adult noncommunicable chronic diseases, it will not be cured but rather "controlled" for a growing number of years, ensuring an ever-improving quality of life, such as we see today with diabetes mellitus or hypertension.

Biotechnology products, especially immunotherapy with monoclonal antibodies and vaccines, can achieve an equally successful therapeutic strategy for advanced malignant neoplasms.[7] For this type of strategy to make an impact on population mortality rates, very long-term chronic treatments need to be tested.

Cancer incidence peaks at about age 67 years. A treatment that extends 5-year survival in a population with a life expectancy of 77 years, would still not modify the mortality rate. Therapeutic results for cancer that we measure today in months of survival gained will need to be expressed in years.
- Long-term control of malignant neoplasms increasingly requires a type of clinical trial that evaluates combinations of products, rather than just single ones. Where chemotherapy has made a major impact on survival (lymphoblastic leukemia, lymphomas, nonseminoma testicular carcinoma, adjuvant breast cancer treatment, among others), it has done so with therapeutic combinations. These were the focus of chemotherapy development during the 1970s and 1980s, when the intellectual property protection environment was much more permissive. Now, the more stringent regulatory context increasingly inhibits scientific research on therapeutic combinations. The publicly-owned Cuban biotechnology industry may have an important advantage when studying therapeutic combinations with products from several of its institutions. Even so, we will need to revise the regulatory context to hasten greater use of this type of trial.

- Clinical research has to be grounded in the "real patient," who, in oncology, is frequently advanced in age and has one or more comorbidities. One of the most worrisome distortions of current therapeutic clinical trial practice in the world is that, in the interest of creating homogenous trial groups and low variability, trials are conducted with ever more strict inclusion criteria, far removed from real patients in everyday medical practice. This leads us to the absurdity of medical research producing therapeutic standards applicable to ever smaller subsets of patients. Thus, recent studies have shown that in real oncological practice, less than $50 \%$ of patients receive what is considered "state-ofthe-art" treatment,[8] while developing optimal therapies for these real patients is not among the research priorities financed by the global pharmaceutical industry.

- One of the many consequences of conducting increasingly longer clinical trials, with less toxic biological products and inclusion criteria that permit participation by ever larger patient subsets, is the need to fully introduce clinical trials into primary health care. In recent decades, therapeutic clinical research has been increasingly concentrated in
Figure 4: Transforming Advanced Cancer into a Chronic Disease

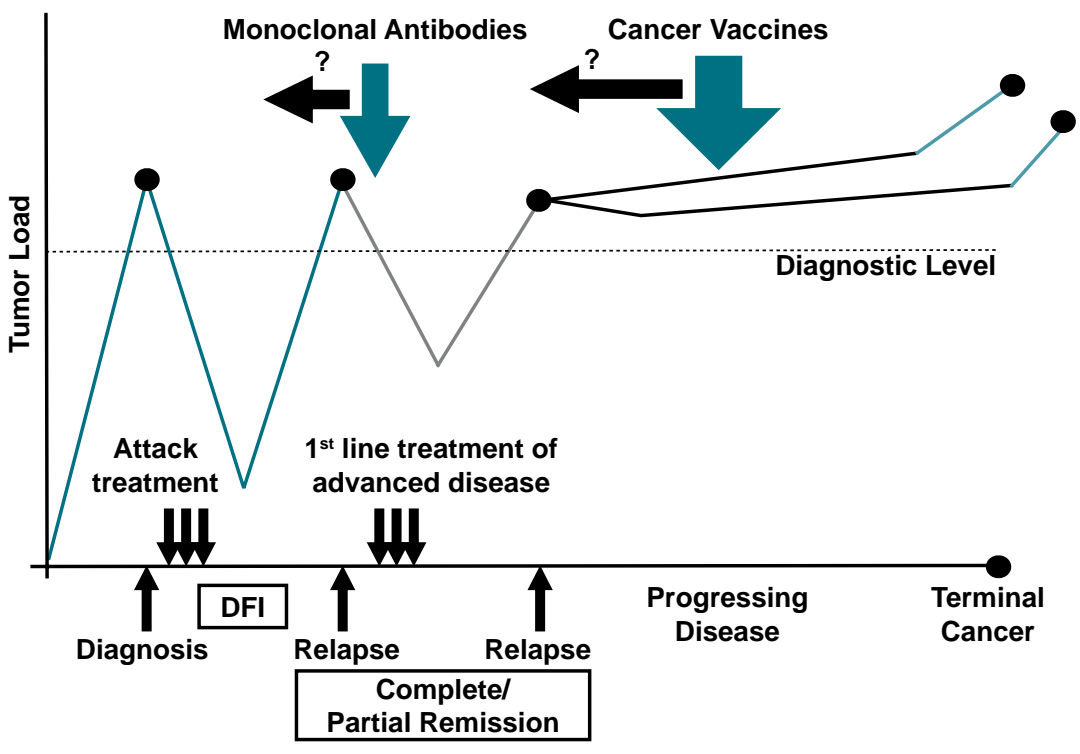

DFI: Disease-Free Interval 


\section{Policy \& Practice}

large institutions that centralize resources and capacities at the top of the health institution pyramid. But it's at the bottom of the pyramid where the battle must be waged for population health impact of medical interventions. We must find organizational formulas for clinical research to be carried out more often in our community-based polyclinics. Biotechnology and basic research must be connected ever more closely to public health.[9]

These ideas and recommendations about chronic treatments, extending clinical trials, evaluating combinations, broadening inclusion criteria, displacing the system's center of gravity toward primary care, and others, clearly challenge the reductionist focus of medical research during the last 50 years. All these would have to be implemented, of course, without sacrificing the objectivity of scientific judgment or "evidence-based" medical decision-making. Such an intellectual and organizational challenge-with no pre-ordained path to the answers-demands above all a systematic and creative approach.

Cuban biotechnology and our National Cancer Program are in an excellent position to take up the challenge.

\section{References \& Notes}

1. Ernst \& Young. Beyond Borders: The Global Biotechnology Report 2007 [monograph on the Internet].OECD; 2007 [cited 2008 Jun]. Available from: http://www.ey.com/Publication/vwLUAssets/beyond_borders2007/\$FILE/ BeyondBorders2007.pdf

2. van Beuzekom B, Arundel A. OECD, 2006. Biotechnology Statistics [monograph on the Internet]. OECD; 2006 [cited 2008 Jun]. Available from: http:// www.oecd.org/dataoecd/51/59/36760212.pdf

3. Pharmaceutical Manufacturers Association of America, 2008. Biotechnology Medicines 2008 [monograph on the Internet]. PhRMA; 2008 [cited 2009 Apr]. Available from: http://www.phrma.org/images/110308\%20biotech\%20 2008.pdf

4. Eisenhauer EA. Response evaluation: beyond RECIST. Ann Oncol. 2007 Jul;18(Suppl 9):ix29-32. Review.

5. Evenson D. Cuba's Biotechnology Revolution. MEDICC Review. 2007 Fall;9(1):8-10.

6. Lage A. Connecting science to population health: the "closed loop" approach. MEDICC Review. 2007 Fall;9(1):48.

7. Lage A, Pérez R, Fernández LE. Therapeutic Cancer Vaccines: at midway between immunology and pharmacology. Curr Cancer Drug Targets. 2005 Dec;5(8):611-27.

8. Earle CC, Tsai JS, Gelber RD, Weinstein MC, Neumann PJ, Weeks JC. Effectiveness of Chemotherapy for Advanced Lung Cancer in the Elderly: Instrumental Variable and Propensity Analysis. J Clin Oncol. 2001 Feb 15;19(4):1064-70.

9. Lage A. Connecting immunology research to public health: Cuban biotechnology. Nat Immunol. 2008 Feb;9(2):109-12. 